\title{
Epithelial-mesenchymal transition in Crohn's disease
}

\author{
H Jiang ${ }^{1}$, J Shen ${ }^{1}$ and Z Ran ${ }^{1}$
}

Crohn's disease (CD) is often accompanied by the complications of intestinal strictures and fistulas. These complications remain obstacles in CD treatment. In recent years, the importance of epithelial-mesenchymal transition in the pathogenesis of CD-associated fistulas and intestinal fibrosis has become apparent. Epithelial-mesenchymal transition refers to a dynamic change, wherein epithelial cells lose their polarity and adherence and acquire migratory function and fibroblast features. During formation of $\mathrm{CD}$-associated fistulas, intestinal epithelial cells dislocate from the basement membrane and migrate to the lining of the fistula tracts, where they convert into transitional cells as a compensatory response under the insufficient wound healing condition. In CD-associated intestinal fibrosis, epithelial-mesenchymal transition may serve as a source of new fibroblasts and consequently lead to overproduction of extracellular matrix. In this review, we present current knowledge of epithelial-mesenchymal transition and its role in the pathogenesis of $C D$ in order to highlight new therapy targets for the associated complications.

\section{INTRODUCTION}

Therapeutic strategies for Crohn's disease (CD) have progressed remarkably in the past decade. ${ }^{1}$ From 1991 to 2014, the rate of hospitalization and surgical intervention for CD decreased; however, despite the use of advanced drugs such as immunomodulators and biologics, the progression to complicated disease forms remained constant. ${ }^{2} \mathrm{CD}$ has traditionally been a problematic condition owing to its associated complications of strictures and fistulizing diseases. Approximately $70 \%$ of patients are affected by fistula and stenosis, and about two-thirds require surgical intervention at least once in the 20 years after primary diagnosis. ${ }^{3}$ However, surgery cannot guarantee a definite solution, and problematic complications reoccur frequently. ${ }^{4}$ Although therapies including antitumor necrosis factor (TNF) antibodies have been developed to control inflammation, and to induce remission, the long-term efficacy of these therapies in preventing $\mathrm{CD}$ progression to fibrosis and fistula is not promising. ${ }^{2,5}$ Further study of the cellular and molecular pathophysiological processes involved in the complicating phenotypes of CD might help identify appropriate treatments to benefit patients with CD.

In recent years, studies have demonstrated that epithelialmesenchymal transition (EMT) plays a crucial role in fibrosis and fistula formation in CD. ${ }^{6,7}$ EMT was first identified in the 1960s from the pioneering work of Elizabeth Hay, who described transformation of epithelial cells to mesenchymal cells in the chick primitive streak during embryogenesis. ${ }^{8}$ Generally, in EMT, epithelial cells demonstrate plasticity and lose epithelial markers like E-cadherin and catenins. Subsequently, other changes occur, such as development of spindleshaped morphology, which is typical of mesenchymal cell lines, and increased extracellular matrix (ECM)-producing capacity. In addition, cells show expression of mesenchymal-related molecular markers such as fibroblast-specific protein-1 (FSP1) and vimentin. ${ }^{9,10}$ Of note is the expression of a powerful inducer of EMT - transforming growth factor- $\beta$ (TGF- $\beta){ }^{11}$

Depending on the biologic conditions, EMT can be categorized into three types: type 1 is present in implantation, embryogenesis, and organ development; type 2 is correlated to tissue regeneration and fibrosis; and type 3 is involved in tumor growth and cancer progression. ${ }^{12}$ Fibrosis is a pathologic response to chronic inflammation and impaired regeneration, and is defined as excess accumulation of ECM, resulting to some extent from the expansion of mesenchymal cells. ${ }^{13,14}$ Wound healing and intestinal fibrosis are inevitable in the disease course of CD. The pathophysiologic effects of EMT in

${ }^{1}$ Division of Gastroenterology and Hepatology, Key Laboratory of Gastroenterology and Hepatology, Ministry of Health, Inflammatory Bowel Disease Research Center; Renji Hospital, School of Medicine, Shanghai Jiao Tong University; Shanghai Institute of Digestive Disease, Shanghai, China. Correspondence: J Shen (shenjun@vip.163.com) 
tissue repair, fibrosis, and fistula formation in disease progression and possible carcinogenic aspects of $\mathrm{CD}$ are emphasized in this review. With a thorough and comprehensive understanding of EMT in CD, early markers for detection and novel drug therapies for strictures and fistula can be developed in tandem with EMT and aid in the current treatment of these complicating phenotypes of $\mathrm{CD}$.

This review aims to present the current knowledge about EMT, highlight its role in fibrosis, fistula formation, and carcinogenesis in $\mathrm{CD}$, and discuss the prospective medical advancements related to EMT in CD.

\section{EPITHELIAL-MESENCHYMAL TRANSITION}

EMT describes a series of changes in which epithelial cells lose some of their epithelial characteristics such as polarity and adhesiveness and acquire properties of mesenchymal cells. Such transitions occur in several biological scenarios and their consequences have attracted the interest of scientists from different fields. The reverse process, called mesenchymalepithelial transition (MET), has also been reported. ${ }^{15}$

The shift from epithelial to mesenchymal cells includes a wide range of inter- and intracellular changes, such as expression of transcription factors, RNAs, TGF- $\beta$ family proteins, and tyrosine kinase receptors; these factors work in different combinations in different types of EMT. ${ }^{16}$ Researchers widely acknowledge the proposal discussed at a 2007 meeting on EMT in Poland and at a subsequent conference in March 2008 at Cold Spring Harbor Laboratories for classifying EMTs into three different subtypes on the basis of the biological settings in which they occur.

\section{EMT in development}

EMT associated with implantation, embryogenesis, and organ development has been defined as type 1 EMT. This type of physiological EMT represents the developmental need to generate various mesenchymal cells that possess the ability to form new organs from epithelial lines. ${ }^{17}$ In the later stages, primary mesenchymal cells acquire the potential to generate secondary epithelial cells with specific functions via MET. ${ }^{18}$

Type 1 EMT occurs as early as during implantation of the embryo into the uterus. The trophectoderm cells undergo EMT to invade the endometrium and, consequently, settle in the placenta. ${ }^{19}$ In gastrulation, a subgroup of epiblast cells from the embryonic single epithelial cell layer move to the midline to form the primitive streak. These cells undergo EMT to produce the mesoderm and endoderm, whereas those that do not undergo EMT form the ectoderm. Thus, the embryo develops from one layer into three germ layers. ${ }^{20,21}$ In addition, primary EMT contributes to the development of the vertebrate nervous system to generate neural crest cells. Epithelial cells originating from the dorsal midline of the neural tube undergo EMT to transform into migratory neural crest cells that later spread out along the embryo and form neural crest mesenchyme; this mesenchyme further differentiates into neurons, glial cells, and many other cell types. ${ }^{22}$ Subsequently, secondary EMT, tertiary

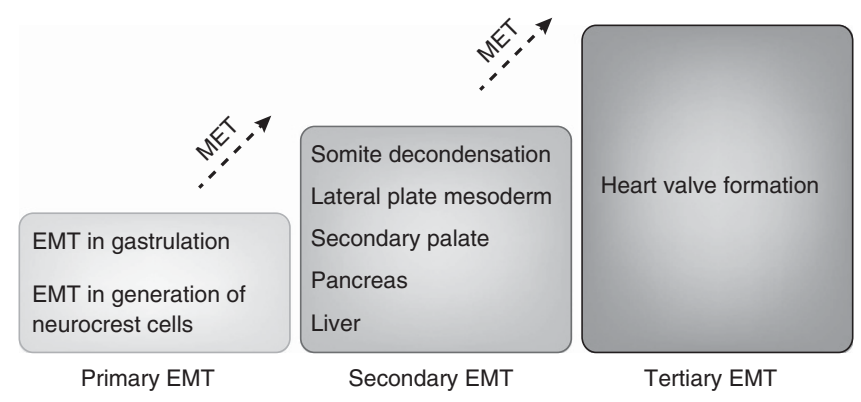

Figure 1 Type 1 EMT in embryogenesis and organ development. Primary EMT occurs in the gastrulation stage, contributing to the generation of mesoderm, endoderm, and neurocrest cells that further develop into neurons, glial cells, and many other cell types. Secondary EMT is involved in the architecture of many organs such as the pancreas and liver. Tertiary EMT is required for heart valve formation. Overall, EMTs and METs participate in embryogenesis and organ formation. EMT, epithelial-mesenchymal transition; MET, mesenchymal-epithelial transition.

EMT, and MET participate sequentially in constructing the final architecture of various organs and tissues (Figure 1). ${ }^{23}$

\section{EMT in tissue repair and fibrosis}

Type 2 EMT occurs in the pathophysiological settings of tissue repair and fibrosis. Such phenotypic changes occur when there is a need to recruit mesenchymal cells from epithelial cells during tissue damage. During skin wound healing, EMT contributes to the differentiation of myofibroblasts that are vital for ECM deposition. ${ }^{24}$ Epithelial cells gain motility through EMT and migrate to the site of injury to trim defects and restore tissue integrity; however, the ECM depositions secreted by the fibroblasts, and partially by epithelial cells via EMT, can result in unintended fibrosis and potential organ dysfunction. ${ }^{25,26}$

Several studies in cultured cells in vitro and in animal models and human specimens in vivo have provided evidence of EMT in fibrogenesis of critical organs such as kidney, liver, lung, and intestine. ${ }^{6,27-29}$ By using a transgenic mouse model, a lineagetagging study provided strong evidence verifying the important role of EMT in renal fibrosis, and reported that $>30 \%$ of new fibroblasts are derived from local EMT. ${ }^{25}$ A similar phenomenon of EMT was reported in the liver and in cases of pulmonary fibrosis. ${ }^{28,29}$ Another in vivo study of human kidney tissues by immunohistochemical tests demonstrated that the type 2 EMT process in humans is regulated by crosstalk among the TGF- $\beta /$ Smad, integrin/integrin-linked kinase, and Wnt $/ \beta$ catenin signaling pathways. ${ }^{30}$ TGF- $\beta$ activates SMAD2 and SMAD3 that translocate to the nucleus to activate transcriptional regulators for repression or activation of target genes. ${ }^{16}$ Mouse Smad3 $3^{-1-}$ models are protected against tubulointerstitial fibrosis, as shown by blockage of the EMT TGF- $\beta$ signals and ECM deposition. ${ }^{31}$ In addition, systemic injection of recombinant human bone morphogenic protein-7, which targets TGF- $\beta$ pathways, can reverse renal fibrosis in mouse models. ${ }^{32,33}$ However, the contribution of EMT to renal fibrosis is controversial. Mack and Yanagita ${ }^{34}$ listed a number of studies describing the origin of myofibroblasts. One of the studies (LeBleu et al..$^{35}$ ) reported that EMT contributes $<5 \%$ to myofibroblasts. Such disputes over the contribution of EMT to 
renal fibrosis should also be noticed. Thus, EMT might not be as important as previously thought, and an exaggerated belief in targeting EMT may be unwise for treatment of fibrosis. The lack of consistent markers for evaluating EMT, tissue heterogeneity, the dynamic features of EMT, and problems with reproducibility in animal models may be responsible for these observed differences between studies.

Nevertheless, pilot and detailed studies of EMT-associated molecules in renal and liver fibrotic diseases have shed light on the role of EMT in intestinal fibrosis. Flier et al., ${ }^{6}$ by using a transgenic mice mouse model for the first time, showed that EMT could contribute to one-third of the FSP1 + fibroblast population in the pathogenesis of intestinal fibrosis. In addition, studies have demonstrated the involvement of EMT in the pathogenesis of fistulas in $\mathrm{CD}^{7,36}$ The disparity in the roles of EMT in renal fibrosis and in intestinal fibrosis of $\mathrm{CD}$ may be explained by the distinct preceding inflammation and microenvironmental factors in CD that differ from those in renal fibrosis. Therefore, a comprehensive understanding of EMT in CD is important.

\section{EMT in tumor progression and metastasis}

In the past two decades, extensive evidence supporting the critical role of EMT in tumor progression, from phenotypic changes to genetic modulation, has been reported. ${ }^{37-39}$ Type 3 EMT is defined as the pathologic process of converting primary tumor epithelial cells into invasive and metastatic mesenchymal tumor cells with better migratory abilities, following which the tumor mesenchyme can leave its original tissue, move within the blood to a distant destination, and convert back into epithelial cells to form new tumor territory through MET. The loss of the epithelial marker E-cadherin and gain of mesenchymal markers such as FSP1 are key indicators of EMT and have been observed in various epithelial cancers such as breast, cervical, ovarian, and colorectal cancer. ${ }^{40}$ Conditions such as hypoxia and chronic inflammation induce cellular changes in unstable genomes that regulate the EMT process and facilitate phenotypic changes in tumor cells. A large amount of evidence identifies the roles of the SMAD/STAT3 signaling

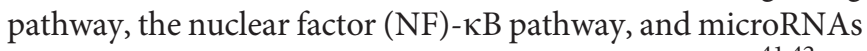
in the development of colorectal cancers via EMT. ${ }^{41,42}$ In addition, studies on the tumor-initiating cells responsible for metastasis showed that breast stem cells associated with generating tumor-initiating cells are under the control of EMT programs that differ from those in physiological regeneration. ${ }^{43,44}$ The role of EMT-independent machinery of tumor invasion, such as podoplanin-mediated actin remodeling, has been identified in some squamous cell carcinomas. ${ }^{45}$ Therefore, EMT alone cannot complete the process of tumor metastasis, nor can all fibrogenesis events occur via EMT.

\section{EMT IN CD}

\section{EMT in CD-associated fistulas}

Fistulas are a problematic complication in CD patients. In a cohort study in Olmsted County, Minnesota, the reported cumulative risk of fistulas was 33\% 10 years after disease onset, with distribution in the perianal (54\%), enteroenteric (24\%), and rectovaginal (9\%) regions, as well as other locations (13\%) such as enterocutaneous, enterovesical, and intraabdominal. ${ }^{46}$ Fistulas are a heavy burden on CD patients and largely decrease their quality of life because of the requirement for surgery and high recurrence rates. ${ }^{3,46}$

Fistula formation may occur as a long-term consequence of chronic inflammation and possibly be driven by EMT. Through EMT, intestinal epithelial cells (IECs) convert into transitional cells (TCs) with mesenchymal myofibroblast-like features and line at the CD fistulas. ${ }^{7,47}$ TCs are a particular kind of cells that are present in the "non-epithelialized" areas of the fistulas; they have mesenchymal features and form an incomplete basement membrane. Such cells occur only in CD fistulas, not in otherwise normal people. TCs lining perianal or enteroenteric fistulas express epithelial markers cytokeratin-8 and cytokeratin-20, but not vimentin, thereby reflecting their epithelial origin. ${ }^{7}$ Expression of the typical epithelial markers E-cadherin and $\beta$-catenin is downregulated in TCs compared with adjacent mucosal epithelial cells and squamous cells. ${ }^{7}$ Reduction in E-cadherin levels is also a feature of EMT. Cadherins are transmembrane proteins facilitating homotypic interaction between neighboring cells to create adherens junctions. ${ }^{48} \beta$ Catenin is the direct physiological link between cadherins and the actin cytoskeleton at adherens junctions. Its nuclear translocation is a determining step of EMT. ${ }^{49}$ Reduction of $\beta$-catenin levels in the cell membrane could allow the cell to acquire migratory abilities. Compared with normal IECs, in which $\beta$-catenin is distributed in the lateral cell membrane, TCs demonstrate a weak, disseminated staining pattern in the cytoplasm, indicating reshaping. ${ }^{7}$ Another key piece of evidence of EMT presence in fistula formation is that $\beta 6$-integrin shows a strong staining pattern in the transitional zone where TCs are present compared with that in normal epithelium. ${ }^{7}$ Integrin complexes enable cells to receive signals from ECM proteins through interactions with signaling mediators such as integrin-linked kinase. ${ }^{16}$ Overexpression of $\beta 6$-integrin is related to the enhancement of cell invasiveness in EMT. ${ }^{37}$ Moreover, previous studies have demonstrated that TGF- $\beta$ is a key inducer of EMT, and immunohistochemistry has shown an elevation in TGF- $\beta 1$ and TGF- $\beta 2$ expression in the fistula-lining cells compared with common IECs. ${ }^{7}$

Although the role of EMT in the pathogenesis of fistulas is remarkable with regard to the changes in cytoskeletal molecules of epithelial cells, the inducers of EMT, that is, TNF and TGF- $\beta$, are also important. Colonic lamina propria fibroblasts (CLPFs) involved in the pathogenesis of $\mathrm{CD}$ fistula are known to have decreased migratory ability and insufficient wound healing when the gut experiences ongoing inflammation. ${ }^{50,51}$ Migration of IECs to the wound area signifies a tendency by employing the epithelial cells to close the defects in a short time. ${ }^{52}$ This migration may be further facilitated by EMT. The reduced migration of CLPFs is caused by the contact of these cells with the proinflammatory molecules interferon- $\gamma$ and TNF. $^{50}$ In addition, TNF has been demonstrated to induce 
EMT in IECs and is capable of inducing TGF- $\beta$ expression. ${ }^{53,54}$ Previous studies on EMT have demonstrated TGF- $\beta$-dependent activation of family members of the SNAIL transcription factor, namely SNAIL1 and SLUG (SNAIL2). ${ }^{55,56}$ The SNAIL transcription factor is a strong repressor of E-cadherin. ${ }^{57} \mathrm{In}$ $\mathrm{CD}$-associated fistulas, SNAIL1 is strongly expressed in the nuclei of TCs lining the fistula tracts with a similar pattern to TGF- $\beta$ expression, indicating increased activity of the TGF- $\beta$ / SNAIL1 pathway. ${ }^{54}$ However, SLUG has limited expression in TCs lining the fistula tracts and is mainly seen in the fibrotic tissue surrounding the fistulas. ${ }^{54}$ Thus, the link between increased TGF- $\beta$ signaling and E-cadherin downregulation can be facilitated by the SNAIL transcription factor. ${ }^{54,57}$

Fistulas may arise because of defective tissue repair, and IECs may reprogram into mesenchymal-like cells whose function is to close wounds. In such scenarios, molecules associated with cell migration and invasion, such as $\beta 6$-integrin, E-twenty-six- 1 (ETS-1) transcription factor, and the Wnt-inhibitor Dickkopfhomolog 1 (DKK-1), have been identified for their role in CD fistulas. $\beta 6$-Integrin is strongly expressed in TCs lining the fistulizing tract. ${ }^{7}$ In addition, researchers observed that TNF induces ETS- 1 and $\beta 6$-integrin expression in HT29 IECs and CLPFs in CD patients with fistulas. ${ }^{53}$ One study on aggressive colon carcinomas found that ETS- 1 was a transcription factor and proto-oncogene that mediates the activation of $\beta 6$-integrin during EMT. ${ }^{37}$ Another inducer of $\beta 6$-integrin is the cytokine interleukin (IL)-13 that mediates the expression of SLUG and $\beta 6$-integrin in HT29 IECs. ${ }^{36}$ Although this in vitro test was done in an EMT model induced by IL-13 treatment, the former part of the same article pointed that IL-13 alone was not sufficient to induce EMT in their IEC models. Proper and replicable EMT models would make it more convincing to determine the molecular features and signaling pathway. More grossly, in fistula samples, high levels of IL-13 were detected in the TCs lining the fistulizing tract. ${ }^{36}$ Compared with healthy controls, TGF- $\beta$ induces IL- 13 secretion in CLPFs of patients with CDassociated fistula. ${ }^{36}$ Furthermore, IL-13 is secreted in an autocrine manner, and both IL-13 and its receptor, -IL$13 \alpha 1$, are highly expressed in cells lining the fistula. ${ }^{36}$ DKK1, a modulator of cell migration and a secreted antagonist of the Wnt signaling pathway, ${ }^{58}$ has higher detectable immunohistochemical staining in TCs lining the fistula tracts in $\mathrm{CD}$ patients than in non-IBD control patients. ${ }^{59}$ Moreover, TGF- $\beta$ treatment increases DKK1 expression in IECs. ${ }^{59}$ A previous study used siRNAs to diminish DDK1 expression in HT-29-IECs in order to study its effect on other genes of fistula pathogenesis; the authors reported that TGF- $\beta$ induces IL-13 expression, but not $\beta 6$-integrin expression, in IECs via DKK-1. ${ }^{59}$

Nucleotide oligomerization domain 2 (NOD2), a receptor for the bacterial cell wall component muramyl dipeptide (MDP), has been found to be associated with the pathogenesis of fistula in $\mathrm{CD} .^{60}$ Further study showed that MDP stimulates expression of TGF- $\beta$, SNAIL1, and IL-13 in IECs and CLPF in fistulas. ${ }^{53}$ These findings suggest that bacteria may play a role in fistula formation in $\mathrm{CD}$ patients, possibly by exogenous induction of EMT.
To summarize the role of EMT in the pathogenesis of CDassociated fistulas (Figure 2), epithelial defects and disturbed immune response to the bacterial component, particularly MDP, leads to a severe inflammatory reaction in the intestinal lumen with the secretion of TNF- $\alpha$, IL-13, and TGF- $\beta$. Subsequently, TGF- $\beta$ induces multiple transcription factors in IECs associated with EMT and cell invasiveness, such as ETS1 , SNAIL1, and SLUG, leading to the repression of E-cadherin, redistribution of $\beta$-catenin, and production of more molecules required for enhanced cell invasiveness, such as $\beta 6$-integrin. IL13 (induced by TGF- $\beta$ ) and its receptor, as well as TNF and its receptors, may act in an autocrine manner to favor EMT and increase its regulation effect on cell migration and penetration into the deeper layers. Other regulatory proteins such as DKK1 may also participate in this process.

\section{EMT in CD-associated intestinal fibrosis}

Intestinal fibrosis with the onset of fibrostenotic strictures is a long-standing complication of $\mathrm{CD}$ and occurs under the influence of multiple triggering factors such as chronic inflammation, bacterial residency, and unbalanced wound healing processes. ${ }^{61-63}$ Strictures are present in $5-10 \%$ of patients when first diagnosed with $\mathrm{CD}$, and $>30 \%$ of patients develop strictures within 10 years of diagnosis. ${ }^{64,65}$ The cumulative rate of surgical intervention in the first 10 years after diagnosis of $\mathrm{CD}$ is $\sim 35-50 \% .^{66,67}$ Moreover, the recurrence rate of $\mathrm{CD}$ is high: $40 \%$ at 4 years after bowel resection, and $50 \%$ at $10-15$ years after ileocecal resection. ${ }^{68}$ Multiple factors have been considered to be involved in the intestinal fibrosis in $\mathrm{CD}$, including cellular and molecular profibrogenic factors, ECM molecules, the matrix metalloproteinase/tissue inhibitor of metalloproteinase system and microbiotic influence, adipose tissues, and genetic and epigenetic modulations. ${ }^{61}$ In the complex network of intestinal fibrosis, EMT is a unique entity that contributes new mesenchymal cells from the epithelium via multiple cellular molecules, such as IL-13 and TGF- $\beta .^{6,69}$ Such a mesenchymal cell pool provides the main effector cells-fibroblasts and myofibroblasts-that synthesize ECM proteins, leading to fibrosis.

Previous studies have found that EMT contributes to 30\% of new FSP1 + fibroblast cells formed from IECs in mouse models of renal and liver fibrosis. ${ }^{6,25,70}$ A previous study used human specimens and mouse models to investigate the mediators and markers of EMT in CD-associated fibrosis, for example, TGF- $\beta 1$, IL-13, and E-cadherin; it showed that TGF- $\beta 1$ staining was stronger in the epithelial layer of human intestinal fibrotic specimens of $\mathrm{CD}$ patients than in the nonIBD controls, with notable TGF- $\beta 1$ expression in the submucosal layer, particularly the fibrotic area. ${ }^{63}$ Moreover, transient transgenetic mouse models have demonstrated that overexpression of TGF- $\beta 1$, which is the main inducer of EMT, can lead to colonic strictures. ${ }^{71}$ Thus, TGF- $\beta 1$ is evidently a key profibrogenic factor in intestinal fibrosis. Another synergetic and highlighted profibrogenic soluble factor is IL-13 that is involved in fistula formation. ${ }^{61}$ To investigate the relationship 


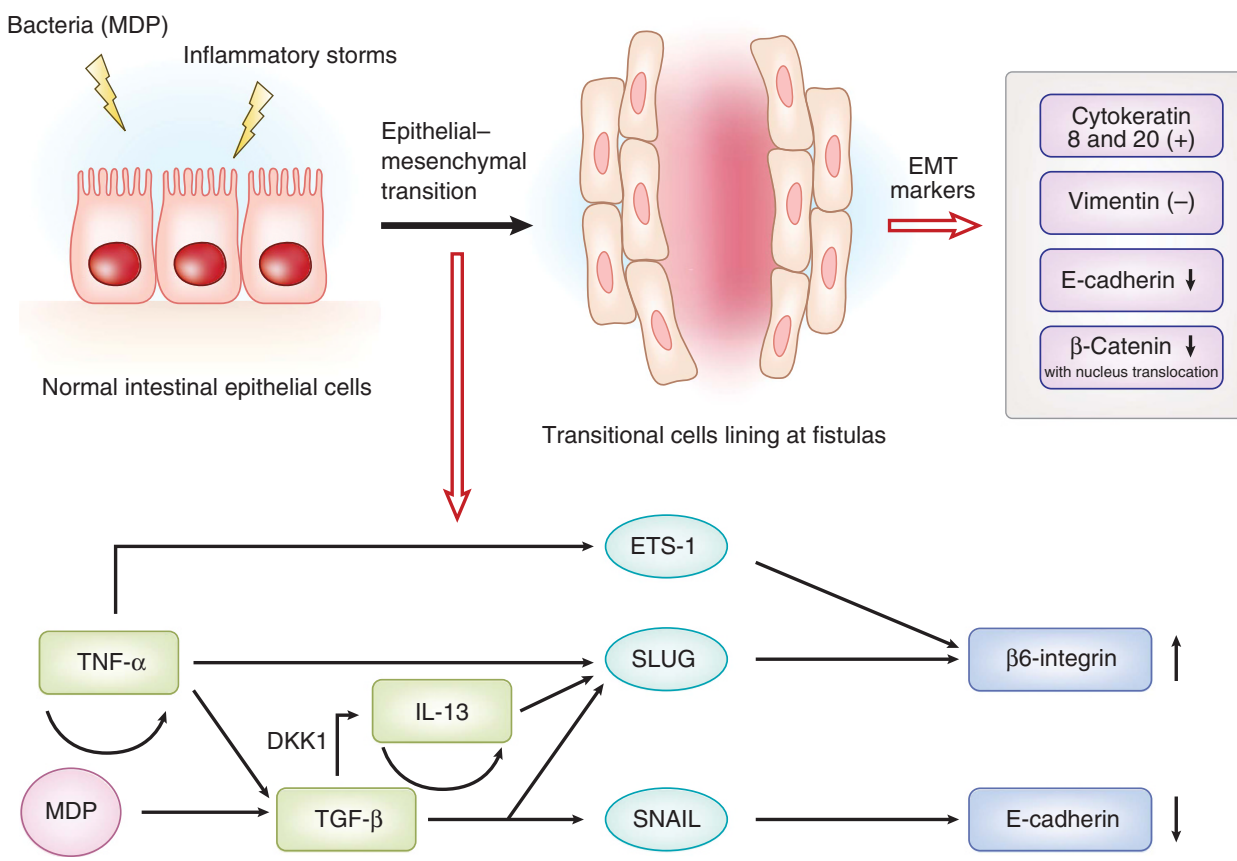

Figure 2 Illustration of EMTs in CD-associated fistula formation. Normal IECs undergo EMT and convert into TCs lining the fistula tract under conditions of epithelial defect and disturbed immune response to bacterial components, particularly MDP. Severe inflammatory reaction in the intestinal lumen presents with the secretion of TNF- $\alpha$, IL-13, and TGF- $\beta$. TGF- $\beta$ induces multiple transcription factors in IECs associated with EMT and cell invasiveness, such as ETS-1, SNAIL1, and SLUG, that leads to the repression of E-cadherin and production of $\beta 6$-integrin. IL- 13 (induced by TGF- $\beta$ ) and its receptor, as well as TNF and its receptors, may act in an autocrine manner to favor EMT. Other regulatory proteins such as DKK1 may also participate in this process. CD, Crohn's disease; DKK, Dickkopf-homolog; EMT, epithelial-mesenchymal transition; ETS, E-twenty-six; IEC, intestinal epithelial cell; IL, interleukin; MDP, muramyl dipeptide; TC, transitional cell; TGF, tissue growth factor; TNF, tumor necrosis factor.

between immune regulators and TGF- $\beta 1$, researchers have used a chronic colitis murine model induced by long-term intrarectal administration of the chemical TNBS $(2,4,6-$ trinitrobenzene sulfonic acid), with the apparent colonic collagen deposition mimicking inflammation-induced colonic fibrosis. ${ }^{69}$ The experiments showed that IL- 13 induces TGF- $\beta 1$ by expression of IL-13R on the cell surface. ${ }^{69}$ Furthermore, $\beta$-catenin, which is correlated with E-cadherin and the cytoskeletalon system as discussed above, was dislocated from the cell membrane and occurred at the perinuclear site, indicating transcriptional activity in the fibroblasts of the fibrotic area of $\mathrm{CD}$ specimens. ${ }^{63}$ As $\beta$-catenin nuclear translocation is an acknowledged feature of EMT, this phenomenon indicates the involvement of EMT in CDassociated fibrosis. Moreover, TGF- $\beta$ has been shown to modulate the expression of SLUG via $\beta$-catenin in epithelial cell activity of the ocular system, and SLUG is known to be involved in the pathogenesis of EMT via repression of E-cadherin expression. ${ }^{72}$ Identification of this Snail family factor in CD specimens and non-IBD controls indicated that strong SLUG signals were present in the nucleus in fibroblast-like cells in fibrotic areas of specimens from CD patients, but SNAIL1 was absent from both $\mathrm{CD}$ and non-IBD control patients. ${ }^{63}$

Recently, studies have reported that microRNAs (miRNAs) mediate EMT and promote fibrotic diseases in many organs, such as lung, kidney, and heart. ${ }^{73}$ MiRNAs are small, noncoding RNAs of 18-25 nucleotides that regulate gene and protein activities at the posttranscriptional level. ${ }^{74}$ With regard to IBD, miRNAs have been found in various pathologic processes, including regulation of the NF- $\kappa \mathrm{B}$ pathway (e.g., miR-146a and miR-146b), intestinal epithelial barrier function (e.g., miR-21 and miR-200b), and autophagy (e.g., miR-30c and miR-130a). ${ }^{75}$ Among these miRNAs, miR-200b has been found to target the zinc-finger E-box-binding homeobox (ZEB) transcriptional factor, and its overexpression could inhibit TGF- $\beta 1$-induced EMT in IECs. ${ }^{76}$ In another study, researchers demonstrated that miR-200b could partially protect IECs from fibrogenesis by suppressing EMT in vitro. ${ }^{77}$ Introduction of miR-200b in TGF- $\beta 1$-induced cell lines causes an increase in the expression of E-cadherin and a reduction in the expression of vimentin, fibronectin, and $\mathrm{N}$-cadherin that contrasts with the pattern seen in intestinal fibrosis in $\mathrm{CD}^{77}$ Therefore, we believe that miR-200b could repress ZEB, ameliorate the effect of TGF- $\beta 1$ in EMT, and prevent intestinal fibrosis in CD. However, more in vivo evidence is required to confirm this hypothesis.

The intestinal microbiota, a critical part of the gut environment, is known to impact IBD pathogenesis and fibrosis. $^{78}$ Microbes contribute to mucosal homeostasis and maintain barrier function. Host-microbe interaction is facilitated by pattern recognition receptors, such as Toll-like receptors and Nod-like receptors, in all types of intestinal cells. ${ }^{79}$ Rieder et al. ${ }^{62,80}$ found that stimulation of Toll-like receptor and Nod-like receptor ligands of the intestinal fibroblasts induced a fibrogenic response independent of TGF- $\beta 1$. Another previous study on microbial lipopolysaccharide 
demonstrated the presence of microbe-induced EMT in intrahepatic biliary epithelial cells. ${ }^{81}$ Comparably, Helicobacter pylori has been found to enhance EMT in gastric cancer by inducing expression of SNAIL and SLUG transcription factors. $^{82}$ A recent study used Young Adult Mouse Colon (YAMC) cells to confirm that EMT events were promoted by bacterial infection. ${ }^{83}$ There is still no direct evidence of microbe-induced EMT in CD specimens; however, the previous results indicate the possibility of microbial involvement. Hofman and Vouret-Craviari ${ }^{84}$ suggested that microbes induced chronic inflammation in a population predisposed to dysbiosis, genetic defects in, for example, NOD2 variants and epigenetic changes that activated the intracellular NF- $\kappa \mathrm{B}$ and MAPK signaling pathways and led to EMT. The microbiome is involved in numerous processes from production of chemicals to genetic modulation, but its correlation with EMT is poorly understood. Therefore, it is important to understand how microbes induce EMT, the relationship between the microbiome and chronic inflammation, and the role of the microbiome in intestinal fibrosis in CD.

In conclusion, existing evidence indicates the involvement of EMT in the fibrogenesis of CD. According to our current knowledge, the possible pathogenesis of CD-associated intestinal fibrosis is as follows: IL-13 induces the expression of TGF$\beta$, and through downstream signaling, $\beta$-catenin undergoes nuclear translocation, thereby activating transcription factors related to EMT. Of these factors, we have identified the SLUG transcription factor. In addition, miRNA-200b has the potential to ameliorate TGF- $\beta 1$-induced EMT by targeting the ZEB transcription factor in intestinal cells, and the microbiome may have a role in triggering EMT in intestinal fibrosis.

\section{EMT in carcinogenesis of CD}

$\mathrm{CD}$ has been reported to have carcinomas of both the small and large bowel as long-term complications. ${ }^{85,86}$ In a cohort study, Jess et al. ${ }^{87}$ reported an increased risk of $>60$-fold for small bowel adenocarcinoma compared with the general population. Regarding colorectal cancer, disease duration, age at diagnosis of $\mathrm{CD}$, distal localization, and penetrating disease have been identified as risk factors. ${ }^{88}$ A study also reported the progression of long-standing fistulas in CD to malignancy. ${ }^{89}$ We have discussed type 3 EMT, which is correlated to tumor progression and metastasis, above. In addition, Scharl et al. ${ }^{90}$ reported a case of EMT in a fistula-associated anal adenocarcinoma of CD. They performed immunohistochemical analysis of the EMTassociated molecules SNAIL 1, SLUG, $\beta$-catenin, and E-cadherin in both fistula and carcinoma tissues. The results demonstrated the presence of SLUG in TCs lining the fistula tract that is peculiar to carcinoma fistulas. The distribution pattern of SNAIL 1, $\beta$-catenin, and E-cadherin in this case was consistent with their previous findings addressed in the previous conterpart. ${ }^{7}$ This pilot study and case report revealed the potential involvement of EMT in the carcinogenesis in CD patients; however, the underlying mechanisms remain unclear and require further investigation.
Table 1 Targeted molecules related to EMT, their blocking drugs, and evidence of therapeutic effects on fistulas and/or fibrosis

\begin{tabular}{lcl}
\hline $\begin{array}{l}\text { Targeted } \\
\text { molecule }\end{array}$ & $\begin{array}{c}\text { Blocking } \\
\text { drug }\end{array}$ & $\begin{array}{c}\text { Evidence of therapeutic effects } \\
\text { on fistulas and/or fibrosis }\end{array}$ \\
\hline TNF & Infliximab & $\begin{array}{l}\text { Anti-TNF joint with thiopurine might be } \\
\text { helpful in the fistula-healing process. }\end{array}$ \\
TGF- $\beta$ & Fresolimumab & $\begin{array}{l}\text { A phase I trial with fresolimumab in human } \\
\text { focal segmental glomerulosclerosis showed } \\
\text { optimistic results. }\end{array}$ \\
& Avotermin & $\begin{array}{l}\text { Avotermin has been involved in phase I/II } \\
\text { trials of dermal scars and was demonstrated } \\
\text { to have effects on reducing the scar size by } \\
\end{array}$ \\
& intradermal injection therapy. ${ }^{116}$
\end{tabular}

B6-Integrin STX-100 STX-100 is currently being examined in a phase II trial in interstitial pulmonary fibrosis patients (ClinicalTrials.gov NCT01371305).

IL-13 Lebrikizumab Lebrikizumab has demonstrated antifibrotic effects in pulmonary fibrotic diseases and showed potent blockage of aberrant lung remodeling. ${ }^{105}$

mTOR Everolimus Everolimus has shown efficacy in CD trials, but its usefulness for treating intestinal fibrosis is under investigation. ${ }^{111}$

CD, Crohn's disease; EMT, epithelial-mesenchymal transition; IL, interleukin; mTOR, mammalian target of rapamycin; TGF, transforming growth factor; TNF, tumor necrosis factor.

\section{THERAPY TARGETS FOR EMT}

To develop effective treatments for fistulizing disease and intestinal strictures, comprehensive knowledge of the complex, diverse, interrelated mechanisms involved in the pathogenesis of these complications is required. Thus far, researchers have investigated multiple agents, including anticytokines, antichemokines, antigrowth factor blockers, Toll-like receptor antagonists, matrix metalloproteinase inhibitors, tissue inhibitor of metalloproteinase promoters, and the use of gene silencing or stem cells to hinder these pathways. ${ }^{13,78,91}$ In this review, we have addressed several cellular molecules involved in EMT for their potential therapeutic applications in CD (Table 1).

Although TNF elevation is observed during fistula formation and intestinal fibrosis, ${ }^{7,69}$ physicians are still skeptical about the effectiveness of its antibody in the treatment of accompanying strictures. With regard to intestinal fibrosis, the evidence for specific use of TNF antagonists as antifibrotic agents (as opposed to anti-inflammatory agents) remains unclear. ${ }^{92}$ Among the four proven anti-TNF agents, only infliximab is indicated for use in fistulizing disease. ${ }^{93}$ In 2014, a global consensus concluded that anti-TNF in combination with thiopurine might be beneficial for healing of fistulas and provide a better closure rate than monotherapy. ${ }^{94}$ As TNF is a major proinflammatory cytokine, it is difficult to distinguish between the effect of the drug on fibrosis or fistula formation, and a reduction in inflammation from the baseline condition. ${ }^{92}$

Extensive evidence indicates that the TGF- $\beta$ signaling pathway is a possible target for treatment of CD complications. 
Table 2 Summary and comparison of EMT in CD-associated fistulas and intestinal fibrosis

\begin{tabular}{|c|c|c|c|c|}
\hline Categories & Evidence & Presumption & Cellular and molecular changes & $\begin{array}{l}\text { Other } \\
\text { regulator }\end{array}$ \\
\hline $\begin{array}{l}\text { EMT in CD- } \\
\text { associated } \\
\text { fistulas }\end{array}$ & $\begin{array}{l}\text { TCs lining the fistulas express epithelial } \\
\text { markers CK8 and CK20, whereas their } \\
\text { E-cadherin and } \beta \text {-catenin expression is } \\
\text { downregulated compared with mucosal } \\
\text { epithelial cells and squamous cells. }{ }^{7} \text { TCs } \\
\text { show reduced } \beta \text {-catenin levels in the cell } \\
\text { membrane, indicating enhanced migra- } \\
\text { tory ability. }{ }^{7} \beta 6 \text {-Integrin shows strong } \\
\text { staining pattern in the transitional zone. }{ }^{7}\end{array}$ & $\begin{array}{l}\text { In fistulizing disease, migratory functions } \\
\text { of myofibroblasts are thought to be } \\
\text { impaired. }{ }^{113} \text { Transformation of epithelial } \\
\text { cells into TCs with enhanced migratory } \\
\text { ability via EMT signifies a tendency to } \\
\text { compensate the failure to close a wound. } \\
113\end{array}$ & $\begin{array}{l}\text { Secretion of TNF- } \alpha \text {, IL-13, and TGF- } \beta \text {. } \\
\text { TGF- } \beta \text { induces transcription factors } \\
\text { associated with EMT, such as ETS- } 1 \text {, } \\
\text { SNAIL } 1 \text {, and SLUG. Repression of } \\
\text { E-cadherin, redistribution of } \beta \text {-catenin, } \\
\text { and production of } \beta 6 \text {-integrin }\end{array}$ & $\begin{array}{l}\mathrm{DKK}^{59} \\
\mathrm{MDP}^{60}\end{array}$ \\
\hline $\begin{array}{l}\text { EMT in CD- } \\
\text { associated } \\
\text { intestinal } \\
\text { fibrosis }\end{array}$ & $\begin{array}{l}\text { EMT contributes to } \sim 30 \% \text { of new } \\
\text { FSP1 + fibroblast cells from IECs. }{ }^{6,70} \\
\text { TGF- } \beta 1 \text { staining is stronger in the } \\
\text { epithelial layer of human intestinal fibrotic } \\
\text { specimens of CD patients. }{ }^{63} \beta \text {-Catenin is } \\
\text { dislocated from the cell membrane and } \\
\text { occurs at the perinuclear site in the } \\
\text { fibroblasts of the fibrotic area in CD } \\
\text { specimens. } 63\end{array}$ & $\begin{array}{l}\text { EMT contributes to the overproliferation } \\
\text { and enhanced migratory ability of myo- } \\
\text { fibroblasts. }{ }^{113} \text { Increasing numbers of } \\
\text { myofibroblasts and excessive ECM } \\
\text { deposition lead to fibrosis and strictures in } \\
\text { CD. }{ }^{113}\end{array}$ & $\begin{array}{l}\text { IL-13 induces the expression of TGF- } \beta \text {. In } \\
\text { downstream signaling, } \beta \text {-catenin under- } \\
\text { goes nuclear translocation. SLUG tran- } \\
\text { scription factor is activated and } \\
\text { modulates cytoskeletal molecules. }\end{array}$ & $\begin{array}{l}\text { miRNA- } \\
200 b^{76} \\
\text { ZEB }^{76}\end{array}$ \\
\hline
\end{tabular}

CD, Crohn's disease; CK, cytokeratin; DKK, Dickkopf-homolog; EMT, epithelial-mesenchymal transition; ETS, E-twenty six; FSP, fibroblast-specific protein; IEC, intestinal epithelial cell; IL, interleukin; MDP, muramyl dipeptide; miRNA, microRNA; TC, transitional cell; TGF, transforming growth factor; TNF, tumor necrosis factor; ZEB, zinc-finger E-box-binding homeobox.

In addition to its role in EMT, this growth factor is crucially involved in processes such as monitoring of cellular responses including cell proliferation, differentiation, proliferation, transformation, and tumor suppression, as well as in regulation of immunological self-tolerance by facilitating regulatory $\mathrm{T}$ cell activation through upregulation of forkhead box P3 (FoxP3). ${ }^{95,96}$ Because of this effect, TGF- $\beta 1$-deficient mice die of severe multi-organ inflammation at a young age. .77,98 $^{9}$ Some clinical trials using the anti-TGF agents fresolimumab and avotermin in organs such as lung, kidney, and skin demonstrated their safety and efficacy. ${ }^{99}$ Another option is to target specific signaling molecules in the TGF- $\beta$ cascade. This approach is more likely to gain success, as it targets specific mediators rather than TGF- $\beta$, a pleiotropic mediator. With regard to the EMT process, $\beta 6$-integrin, a downstream molecule controlled by TGF- $\beta$, is upregulated in various fibrotic disorders, and its blockade is effective for treatment of pulmonary fibrosis and liver fibrosis. ${ }^{100,101}$ Inhibitors of $\beta 6$-integrin significantly downregulate the levels of profibrogenic transcripts and consequently hinder fibrosis through local TGF- $\beta$ inhibition without interfering with the homeostatic role of TGF- $\beta .^{102}$ However, the approach of blocking $\beta 6$-integrin for the treatment of $\mathrm{CD}$ requires further research.

Another molecule-IL-13-involved in both fistula formation and intestinal fibrosis is thought to be a promising therapeutic target. IL-13 is produced by immune cells at the initiation of fibrosis in the intestine and increases the production of TGF- $\beta 1$ in macrophages. ${ }^{69,103}$ Antagonism of IL-13 has been shown to be effective in preventing fibrosis in colitis models. ${ }^{103}$ Furthermore, several IL-13 antibodies, such as lebrikizumab and tralokinumab, were tested in clinical trials for their antifibrotic effects in pulmonary fibrotic diseases and showed potent blockage of aberrant lung remodeling. ${ }^{104,105} \mathrm{In}$ fistulizing disease, the IL-13 antibody demonstrates the ability to suppress IL-13-induced SLUG expression that is involved in enhancement of cell invasiveness and EMT. ${ }^{36}$ However, its therapeutic use is yet to be verified in animal models. Another promising therapy to reduce IL-13 expression is the mammalian target of rapamycin (mTOR) protein inhibitor. In the antifibrotic process, mTOR inhibitors decrease the number of fibroblasts and myofibroblasts and repress profibrogenic cytokine expression, including that of IL- 13 and TGF- $\beta 1$, as well as that of type I and III collagen. ${ }^{106,107}$ mTOR inhibitors have been effective in various fibrotic pathologic conditions. ${ }^{108,109}$ Moreover, an mTOR drug, Everolimus, has shown efficacy in CD trials and its potency against intestinal fibrosis is under investigation. ${ }^{110-112}$

\section{CONCLUSIONS AND PERSPECTIVES}

EMT is a unique biologic process that imparts epithelial cells with enhanced migratory ability and provides new fibroblasts to the mesenchymal cell pool under stimulation. This review has focused on highlighting the role of EMT in CD-associated fistula formation and intestinal fibrosis. The migratory functions of myofibroblasts are thought to be impaired in fistulizing disease, and failure to close a wound is compensated by transformation of epithelial cells into TCs via the EMT and their penetration into deep layers; however, these changes also lead to unwanted fistulas. ${ }^{113}$ On the other hand, overproliferation and enhanced migratory ability of myofibroblasts and excess ECM deposition lead to fibrosis and strictures in CD. ${ }^{113}$ A brief summary and comparison of EMT in CD-associated fistulas and intestinal fibrosis is given in Table 2. Despite extensive evidence from human specimens and in vivo tests, EMT remains a controversial concept. Further functional observations and dynamic experiments are needed to confirm 
and illustrate its contribution to the pathogenesis of CD. Future studies should use appropriate experimental models to mimic the conditions of $C D$ and increase the predictive value of comparable responses to new therapy in clinical practice. Recently, Hahn et al. ${ }^{114}$ provided researchers with a newly generated organoid-based EMT model from intestinal epithelial organoids. By using this model, they verified that TNF- $\alpha$ and TGF- $\beta$ promoted the process of EMT in intestinal epithelial organoids. ${ }^{114}$ In addition, we noted that molecules involved in EMT, such as integrin, IL-13, and TGF- $\beta$, are also involved in the pathogenesis of different conditions and in crosstalk with many other pathways. Furthermore, EMT shows different expression patterns in fistula and fibrosis. Further studies are required to obtain a deeper understanding of the role of EMT in the pathogenesis of CD-associated fistula and fibrosis. In addition, it may be beneficial to identify the highrisk population and stratify the disease activity according to the EMT pathways for better treatment and management. We believe further research into EMT could provide ideas for novel therapeutic targets for these complications of $\mathrm{CD}$.

\section{ACKNOWLEDGMENTS}

This work was supported by the National Science Foundation of China (grant number 81470820).

\section{AUTHOR CONTRIBUTIONS}

H.J. drafted the article and J.S. and Z.R. revised it critically for intellectual content.

\section{DISCLOSURE}

The authors declared no conflict of interest.

c) 2018 Society for Mucosal Immunology

\section{REFERENCES}

1. Talley, N.J. et al. An evidence-based systematic review on medical therapies for inflammatory bowel disease. Am. J. Gastroenterol. 106 ((Suppl 1)), S2-25. quiz S26 (2011).

2. Jeuring, S.F. et al. Improvements in the long-term outcome of Crohn's disease over the past two decades and the relation to changes in medical management: results from the population-based IBDSL cohort. Am. J. Gastroenterol. 112, 325-336 (2016).

3. Bernstein, C.N., Loftus, E.V. Jr., Ng, S.C., Lakatos, P.L. \& Moum, B. Epidemiology and Natural History Task Force of the International Organization for the Study of Inflammatory Bowel Disease (IOIBD). Hospitalisations and surgery in Crohn's disease. Gut 61, 622-629.

4. Mowat, C. et al. Guidelines for the management of inflammatory bowel disease in adults. Gut 60, 571-607 (2011).

5. Uchino, M. et al. Long-term efficacy of infliximab maintenance therapy for perianal Crohn's disease. World J. Gastroenterol. 17, 1174-1179 (2011).

6. Flier, S.N., Tanjore, H., Kokkotou, E.G., Sugimoto, H., Zeisberg, M. \& Kalluri, R. Identification of epithelial to mesenchymal transition as a novel source of fibroblasts in intestinal fibrosis. J. Biol. Chem. 285, 2020220212 (2010).

7. Bataille, F. et al. Evidence for a role of epithelial mesenchymal transition during pathogenesis of fistulae in Crohn's disease. Inflamm. Bowel Dis. 14, 1514-1527 (2008).

8. Hay, E.D. Organization and fine structure of epithelium and mesenchyme in the developing chick embryo. In: Fleischmajer, R \& Billingham, RE, (eds). Epithelial-Mesenchymal Interactions. Williams and Wilkins, Baltimore, MD, 1968. pp 31-35.

9. Mendez, M.G., Kojima, S. \& Goldman, R.D. Vimentin induces changes in cell shape, motility, and adhesion during the epithelial to mesenchymal transition. FASEB J. 24, 1838-1851 (2010).
10. Okada, H., Danoff, T.M., Kalluri, R. \& Neilson, E.G. Early role of Fsp1 in epithelial-mesenchymal transformation. Am. J. Physiol. 273 (4 Pt 2), F563-F574 (1997).

11. Xu, J., Lamouille, S. \& Derynck, R. TGF-beta-induced epithelial to mesenchymal transition. Cell Res. 19, 156-172 (2009).

12. Kalluri, R. \& Weinberg, R.A. The basics of epithelial-mesenchymal transition. J. Clin. Invest. 119, 1420-1428 (2009).

13. Latella, G. et al. Pathophysiology of intestinal fibrosis in IBD. J. Crohns Colitis 8, 1147-1165 (2014).

14. Wynn, T.A. \& Ramalingam, T.R. Mechanisms of fibrosis: therapeutic translation for fibrotic disease. Nat. Med. 18, 1028-1040 (2012).

15. Chaffer, C.L., Brennan, J.P., Slavin, J.L., Blick, T., Thompson, E.W. \& Williams, E.D. Mesenchymal-to-epithelial transition facilitates bladder cancer metastasis: role of fibroblast growth factor receptor-2. Cancer Res. 66, 11271-11278 (2006).

16. Lamouille, S., Xu, J. \& Derynck, R. Molecular mechanisms of epithelialmesenchymal transition. Nat. Rev. Mol. Cell Biol. 15, 178-196 (2014).

17. Hay, E.D. The mesenchymal cell, its role in the embryo, and the remarkable signaling mechanisms that create it. Dev. Dyn. 233, 706-720 (2005).

18. Perez-Pomares, J.M. \& Munoz-Chapuli, R. Epithelial-mesenchymal transitions: a mesodermal cell strategy for evolutive innovation in Metazoans. Anat. Rec. 268, 343-351 (2002).

19. Bischof, P. et al. Implantation of the human embryo: research lines and models. From the implantation research network 'Fruitful'. Gynecol. Obstet. Invest. 62, 206-216 (2006).

20. Ohta, S., Suzuki, K., Tachibana, K., Tanaka, H. \& Yamada, G. Cessation of gastrulation is mediated by suppression of epithelial-mesenchymal transition at the ventral ectodermal ridge. Development 134, 43154324 (2007).

21. Shook, D. \& Keller, R. Mechanisms, mechanics and function of epithelial-mesenchymal transitions in early development. Mech. Dev. 120, 1351-1383 (2003).

22. Bronner, M.E. Formation and migration of neural crest cells in the vertebrate embryo. Histochem. Cell Biol. 138, 179-186 (2012).

23. Acloque, H., Adams, M.S., Fishwick, K., Bronner-Fraser, M. \& Nieto, M.A. Epithelial-mesenchymal transitions: the importance of changing cell state in development and disease. J. Clin. Invest. 119, 1438-1449 (2009).

24. Yan, C. et al. Epithelial to mesenchymal transition in human skin wound healing is induced by tumor necrosis factor-alpha through bone morphogenic protein-2. Am. J. Pathol. 176, 2247-2258 (2010).

25. Iwano, M., Plieth, D., Danoff, T.M., Xue, C., Okada, H. \& Neilson, E.G. Evidence that fibroblasts derive from epithelium during tissue fibrosis. J. Clin. Invest. 110, 341-350 (2002).

26. Liu, Y. Epithelial to mesenchymal transition in renal fibrogenesis: pathologic significance, molecular mechanism, and therapeutic intervention. J. Am. Soc. Nephrol. 15, 1-12 (2004).

27. Jinde, K. et al. Tubular phenotypic change in progressive tubulointerstitial fibrosis in human glomerulonephritis. Am J Kidney Dis 38, 761-769 (2001).

28. Chilosi, M. et al. Aberrant Wnt/beta-catenin pathway activation in idiopathic pulmonary fibrosis. Am. J. Pathol. 162, 1495-1502 (2003).

29. Zeisberg, M. et al. Fibroblasts derive from hepatocytes in liver fibrosis via epithelial to mesenchymal transition. J. Biol. Chem. 282, 23337-23347 (2007).

30. Kim, M.K. et al. The differential expression of TGF-beta1, ILK and wnt signaling inducing epithelial to mesenchymal transition in human renal fibrogenesis: an immunohistochemical study. Int. J. Clin. Exp. Pathol. 6, 1747-1758 (2013).

31. Sato, M., Muragaki, Y., Saika, S., Roberts, A.B. \& Ooshima, A. Targeted disruption of TGF-beta1/Smad3 signaling protects against renal tubulointerstitial fibrosis induced by unilateral ureteral obstruction. J. Clin. Invest. 112, 1486-1494 (2003).

32. Zeisberg, M. et al. Bone morphogenic protein-7 inhibits progression of chronic renal fibrosis associated with two genetic mouse models. Am. J. Physiol. Renal Physiol. 285, F1060-F1067 (2003).

33. Zeisberg, M. et al. BMP-7 counteracts TGF-beta1-induced epithelial-tomesenchymal transition and reverses chronic renal injury. Nat. Med. 9 , 964-968 (2003).

34. Mack, M. \& Yanagita, M. Origin of myofibroblasts and cellular events triggering fibrosis. Kidney Int. 87, 297-307 (2015). 
35. LeBleu, V.S. et al. Origin and function of myofibroblasts in kidney fibrosis. Nat. Med. 19, 1047-1053 (2013).

36. Scharl, M. et al. Interleukin-13 and transforming growth factor beta synergise in the pathogenesis of human intestinal fistulae. Gut 62, 63-72 (2013).

37. Bates, R.C. et al. Transcriptional activation of integrin beta6 during the epithelial-mesenchymal transition defines a novel prognostic indicator of aggressive colon carcinoma. J. Clin. Invest. 115, 339-347 (2005).

38. Moody, S.E. et al. The transcriptional repressor Snail promotes mammary tumor recurrence. Cancer Cell 8, 197-209 (2005).

39. Wang, Y., Dong, X., Hu, B., Wang, X.J., Wang, Q. \& Wang, W.L. The effects of Micro-429 on inhibition of cervical cancer cells through targeting ZEB1 and CRKL. Biomed. Pharmacother. 80, 311-321 (2016).

40. Thiery, J.P. Epithelial-mesenchymal transitions in tumour progression. Nat. Rev. Cancer 2, 442-454 (2002).

41. Lee, $\mathrm{H}$. et al. Persistently activated Stat3 maintains constitutive NF-kappaB activity in tumors. Cancer Cell 15, 283-293 (2009).

42. Burk, U. et al. A reciprocal repression between ZEB1 and members of the miR-200 family promotes EMT and invasion in cancer cells. EMBO Rep. $\mathbf{9}$ 582-589 (2008).

43. Guo, W. et al. Slug and Sox9 cooperatively determine the mammary stem cell state. Cell 148, 1015-1028 (2012).

44. Ye, X. et al. Distinct EMT programs control normal mammary stem cells and tumour-initiating cells. Nature 525, 256-260 (2015).

45. Wicki, A., Lehembre, F., Wick, N., Hantusch, B., Kerjaschki, D. \& Christofori, G. Tumor invasion in the absence of epithelial-mesenchymal transition: podoplanin-mediated remodeling of the actin cytoskeleton. Cancer Cell 9, 261-272 (2006).

46. Schwartz, D.A. et al. The natural history of fistulizing Crohn's disease in Olmsted County, Minnesota. Gastroenterology 122, 875-880 (2002).

47. Bataille, F. et al. Morphological characterisation of Crohn's disease fistulae. Gut 53, 1314-1321 (2004).

48. Leckband, D. \& Sivasankar, S. Cadherin recognition and adhesion. Curr. Opin. Cell Biol. 24, 620-627 (2012).

49. Kalluri, R. \& Neilson, E.G. Epithelial-mesenchymal transition and its implications for fibrosis. J. Clin. Invest. 112, 1776-1784 (2003).

50. Leeb, S.N. et al. Reduced migration of fibroblasts in inflammatory bowel disease: role of inflammatory mediators and focal adhesion kinase. Gastroenterology 125, 1341-1354 (2003).

51. Leeb, S.N., Vogl, D., Falk, W., Scholmerich, J., Rogler, G. \& Gelbmann, C.M. Regulation of migration of human colonic myofibroblasts. Growth Factors 20, 81-91 (2002).

52. Podolsky, D.K. Healing the epithelium: solving the problem from two sides. J. Gastroenterol. 32, 122-126 (1997).

53. Frei, S.M. et al. A role for tumor necrosis factor and bacterial antigens in the pathogenesis of Crohn's disease-associated fistulae. Inflamm. Bowel Dis. 19, 2878-2887 (2013).

54. Scharl, M. et al. Potential role for SNAIL family transcription factors in the etiology of Crohn's disease-associated fistulae. Inflamm. Bowel Dis. 17, 1907-1916 (2011).

55. Peinado, H., Quintanilla, M. \& Cano, A. Transforming growth factor beta-1 induces snail transcription factor in epithelial cell lines: mechanisms for epithelial mesenchymal transitions. J. Biol. Chem. 278, 21113-21123 (2003).

56. Zheng, G. et al. Disruption of E-cadherin by matrix metalloproteinase directly mediates epithelial-mesenchymal transition downstream of transforming growth factor-beta1 in renal tubular epithelial cells. Am. J. Pathol. 175, 580-591 (2009).

57. Vincent, T. et al. A SNAIL1-SMAD3/4 transcriptional repressor complex promotes TGF-beta mediated epithelial-mesenchymal transition. Nat. Cell Biol. 11, 943-950 (2009).

58. Koch, S. et al. Dkk-1 inhibits intestinal epithelial cell migration by attenuating directional polarization of leading edge cells. Mol. Biol. Cell 20, 4816-4825 (2009).

59. Frei, S.M. et al. The role for dickkopf-homolog-1 in the pathogenesis of Crohn's disease-associated fistulae. PLOS ONE 8, e78882 (2013).

60. Radlmayr, M., Torok, H.P., Martin, K. \& Folwaczny, C. The c-insertion mutation of the NOD2 gene is associated with fistulizing and fibrostenotic phenotypes in Crohn's disease. Gastroenterology 122, 2091-2092 (2002).

61. Rieder, F. \& Fiocchi, C. Intestinal fibrosis in IBD-a dynamic, multifactorial process. Nat. Rev. Gastroenterol. Hepatol. 6, 228-235 (2009).
62. Rieder, F. The gut microbiome in intestinal fibrosis: environmental protector or provocateur?. Sci. Transl. Med. 5, 190ps110 (2013).

63. Scharl, M., Huber, N., Lang, S., Furst, A., Jehle, E. \& Rogler, G. Hallmarks of epithelial to mesenchymal transition are detectable in Crohn's disease associated intestinal fibrosis. Clin. Transl. Med. 4, 1 (2015).

64. Louis, E., Collard, A., Oger, A.F., Degroote, E., Aboul Nasr, E.I., Yafi, F.A. \& Belaiche, J. Behaviour of Crohn's disease according to the Vienna classification: changing pattern over the course of the disease. Gut 49, 777-782 (2001).

65. Thia, K.T., Sandborn, W.J., Harmsen, W.S., Zinsmeister, A.R. \& Loftus, E.V. Jr Risk factors associated with progression to intestinal complications of Crohn's disease in a population-based cohort. Gastroenterology 139, 1147-1155 (2010).

66. Solberg, I.C. et al. Clinical course in Crohn's disease: results of a Norwegian population-based ten-year follow-up study. Clin. Gastroenterol. Hepatol. 5, 1430-1438 (2007).

67. Biroulet, L.P. et al. S1184 Cumulative incidence of and risk factors for major abdominal surgery in a population-based cohort of Crohn's disease. Gastroenterology 138, S-199 (2010).

68. Gasparetto, M., Angriman, I. \& Guariso, G. The multidisciplinary health care team in the management of stenosis in Crohn's disease. J. Multidiscip. Healthc. 8, 167-179 (2015).

69. Fichtner-Feigl, S. et al. Induction of IL-13 triggers TGF-beta1-dependent tissue fibrosis in chronic 2,4,6-trinitrobenzene sulfonic acid colitis. J. Immunol. 178, 5859-5870 (2007).

70. Zeisberg, M. et al. Fibroblasts derive from hepatocytes in liver fibrosis via epithelial to mesenchymal transition. J. Biol. Chem. 282, 23337-23347 (2007).

71. Vallance, B.A. et al. TGF-beta1 gene transfer to the mouse colon leads to intestinal fibrosis. Am. J. Physiol. Gastrointest. Liver Physiol. 289, G116-G128 (2005).

72. Choi, J., Park, S.Y. \& Joo, C.K. Transforming growth factor-beta1 represses $\mathrm{E}$-cadherin production via slug expression in lens epithelial cells. Invest. Ophthalmol. Vis. Sci. 48, 2708-2718 (2007).

73. Zou, X.Z., Liu, T., Gong, Z.C., Hu, C.P. \& Zhang, Z. MicroRNAs-mediated epithelial-mesenchymal transition in fibrotic diseases. Eur. J. Pharmacol. 796, 190-206 (2017).

74. Winter, J., Jung, S., Keller, S., Gregory, R.I. \& Diederichs, S. Many roads to maturity: microRNA biogenesis pathways and their regulation. Nat. Cell Biol. 11, 228-234 (2009).

75. Chen, W.X., Ren, L.H. \& Shi, R.H. Implication of miRNAs for inflammatory bowel disease treatment: systematic review. World J. Gastrointest. Pathophysiol. 5, 63-70 (2014).

76. Chen, Y. et al. miR-200b inhibits TGF-beta1-induced epithelial-mesenchymal transition and promotes growth of intestinal epithelial cells. Cell Death Dis. 4, e541 (2013).

77. Chen, Y. et al. miR-200b is involved in intestinal fibrosis of Crohn's disease. Int. J. Mol. Med. 29, 601-606 (2012).

78. Bettenworth, D. \& Rieder, F. Pathogenesis of intestinal fibrosis in inflammatory bowel disease and perspectives for therapeutic implication. Dig. Dis. 35, 25-31 (2017).

79. Takeuchi, O. \& Akira, S. Pattern recognition receptors and inflammation. Cell 140, 805-820 (2010).

80. Rieder, F. et al. Pro-fibrogenic activity of Toll-like receptor (TLR) and NODlike receptor (NLR) ligands on human intestinal myofibroblasts (HIF) linking bacterial innate immunity to intestinal fibrosis. Gastroenterology 138, S-35-S-35 (2010).

81. Zhao, L., Yang, R., Cheng, L., Wang, M., Jiang, Y. \& Wang, S. LPSinduced epithelial-mesenchymal transition of intrahepatic biliary epithelial cells. J. Surg. Res. 171, 819-825 (2011).

82. Yin, Y. et al. Helicobacter pylori potentiates epithelial:mesenchymal transition in gastric cancer: links to soluble HB-EGF, gastrin and matrix metalloproteinase-7. Gut 59, 1037-1045 (2010).

83. Chandrakesan, P. et al. Utility of a bacterial infection model to study epithelial-mesenchymal transition, mesenchymal-epithelial transition or tumorigenesis. Oncogene 33, 2639-2654 (2014).

84. Hofman, P. \& Vouret-Craviari, V. Microbes-induced EMT at the crossroad of inflammation and cancer. Gut Microbes 3, 176-185 (2012).

85. Kerber, G.W. \& Frank, P.H. Carcinoma of the small intestine and colon as a complication of Crohn disease: radiologic manifestations. Radiology 150 , 639-645 (1984). 
86. Ribeiro, M.B. et al. Colorectal adenocarcinoma in Crohn's disease. Ann. Surg. 223, 186-193 (1996).

87. Jess, T., Winther, K.V., Munkholm, P., Langholz, E. \& Binder, V. Intestinal and extra-intestinal cancer in Crohn's disease: follow-up of a populationbased cohort in Copenhagen County, Denmark. Aliment. Pharmacol. Ther. 19, 287-293 (2004).

88. Scaringi, S. et al. Colorectal cancer and Crohn's colitis: clinical implications from 313 surgical patients. World J. Surg. 37, 902-910 (2013).

89. lesalnieks, I. et al. Fistula-associated anal adenocarcinoma in Crohn's disease. Inflamm. Bowel Dis. 16, 1643-1648 (2010).

90. Scharl, M., Frei, P., Frei, S.M., Biedermann, L., Weber, A. \& Rogler, G. Epithelial-to-mesenchymal transition in a fistula-associated anal adenocarcinoma in a patient with long-standing Crohn's disease. Eur. J. Gastroenterol. Hepatol. 26, 114-118 (2014).

91. Scharl, M. \& Rogler, G. Pathophysiology of fistula formation in Crohn's disease. World J. Gastrointest. Pathophysiol. 5, 205-212 (2014).

92. Jacob, N., Targan, S.R. \& Shih, D.Q. Cytokine and anti-cytokine therapies in prevention or treatment of fibrosis in IBD. United European Gastroenterol. J. 4, 531-540 (2016).

93. Danese, S., Vuitton, L. \& Peyrin-Biroulet, L. Biologic agents for IBD: practical insights. Nat. Rev. Gastroenterol. Hepatol. 12, 537-545 (2015).

94. Gecse, K.B. et al. A global consensus on the classification, diagnosis and multidisciplinary treatment of perianal fistulising Crohn's disease. Gut 63, 1381-1392 (2014).

95. Akhurst, R.J. \& Hata, A. Targeting the TGFbeta signalling pathway in disease. Nat. Rev. Drug Discov. 11, 790-811 (2012).

96. Zhou, L. et al. TGF-beta-induced Foxp3 inhibits $T(H) 17$ cell differentiation by antagonizing RORgammat function. Nature 453, 236-240 (2008).

97. Diebold, R.J. et al. Early-onset multifocal inflammation in the transforming growth factor beta 1-null mouse is lymphocyte mediated. Proc. Natl. Acad. Sci. USA 92, 12215-12219 (1995).

98. Kulkarni, A.B. Transforming growth factor-beta 1 null mice. An animal model for inflammatory disorders. Am. J. Pathol. 146, 264-275 (1995).

99. Bettenworth, D. \& Rieder, F. Medical therapy of stricturing Crohn's disease: what the gut can learn from other organs - a systematic review. Fibrogenesis Tissue Repair 7, 5 (2014).

100. Sullivan, B.P., Weinreb, P.H., Violette, S.M. \& Luyendyk, J.P. The coagulation system contributes to $\alpha \vee \beta 6$ integrin expression and liver fibrosis induced by cholestasis. Am. J. Pathol. 177, 2837-2849 (2010).

101. Horan, G.S. et al. Partial inhibition of integrin alpha(v)beta6 prevents pulmonary fibrosis without exacerbating inflammation. Am. J. Respir. Crit. Care Med. 177, 56-65 (2008).

102. Madala, S.K. et al. Inhibition of the alphavbeta6 integrin leads to limited alteration of TGF-alpha-induced pulmonary fibrosis. Am. J. Physiol. Lung Cell. Mol. Physiol. 306, L726-L735 (2014).
103. Fichtner-Feigl, S., Strober, W., Kawakami, K., Puri, R.K. \& Kitani, A. IL-13 signaling through the IL-13alpha2 receptor is involved in induction of TGFbeta1 production and fibrosis. Nat. Med. 12, 99-106 (2006).

104. Murray, L.A. et al. Targeting interleukin-13 with tralokinumab attenuates lung fibrosis and epithelial damage in a humanized SCID idiopathic pulmonary fibrosis model. Am. J. Respir. Cell Mol. Biol. 50, 985-994 (2014).

105. Hanania, N.A. et al. Efficacy and safety of lebrikizumab in patients with uncontrolled asthma (LAVOLTA I and LAVOLTA II): replicate, phase 3, randomised, double-blind, placebo-controlled trials. Lancet Respir. Med. 4, 781-796 (2016).

106. Osman, B., Els, A., Doller, A., Müller, R., Pfeilschifter, J. \& Eberhardt, W. Differential modulation of the cytokine-induced MMP-9/TIMP-1 protease-antiprotease system by the mTOR inhibitor rapamycin. Biochem. Pharmacol. 81, 134-143 (2011).

107. Wang, S. Noncanonical TGF-beta pathways, mTORC1 and Abl, in renal interstitial fibrogenesis. Am. J. Physiol. Renal Physiol. 298, F142-F149 (2009).

108. Messina, M., Ariaudo, C., Mella, A., Cantaluppi, V., Segoloni, G.P. \& Biancone, L. mTOR inhibitors for medical treatment of post-transplantation encapsulating peritoneal sclerosis: a favourable single center experience. J. Nephrol. 28, 245-249 (2015).

109. Syed, F. et al. Keloid disease can be inhibited by antagonizing excessive mTOR signaling with a novel dual TORC1/2 inhibitor. Am. J. Pathol. 181, 1642-1658 (2012).

110. Dumortier, J. et al. Everolimus for refractory Crohn's disease: a case report. Inflamm. Bowel Dis. 14, 874-877 (2008).

111. Reinisch, W. et al. A multicenter, randomized, double-blind trial of everolimus versus azathioprine and placebo to maintain steroid-induced remission in patients with moderate-to-severe active Crohn's disease. Am. J. Gastroenterol. 103, 2284-2292 (2008).

112. Massey, D.C., Bredin, F. \& Parkes, M. Use of sirolimus (rapamycin) to treat refractory Crohn's disease. Gut 57, 1294-1296 (2008).

113. Meier, J.K. et al. Specific differences in migratory function of myofibroblasts isolated from Crohn's disease fistulae and strictures. Inflamm. Bowel Dis. 17, 202-212 (2011).

114. Hahn, S. et al. Organoid-based epithelial to mesenchymal transition (OEMT) model: from an intestinal fibrosis perspective. Sci. Rep. 7, 2435 (2017).

115. Trachtman, H. et al. A phase 1, single-dose study of fresolimumab, an anti-TGF-B antibody, in treatment-resistant primary focal segmental glomerulosclerosis. Kidney Int. 79, 1236-1243 (2011).

116. Ferguson, M.W. et al. Prophylactic administration of avotermin for improvement of skin scarring: three double-blind, placebo-controlled, phase I/II studies. Lancet 373, 1264-1274 (2009). 